\title{
Research on High-Speed Data Acquisition and Real-Time Signal Processing Method Based on Tunable Diode Laser Spectroscopy
}

\author{
X.F. Du \\ National Engineering Research Center for Information \\ Technology in Agriculture \\ Beijing Academy of Agriculture and Forestry Sciences \\ Beijing, China \\ Laboratory of Digital Media and Chip, \\ School of Software and Microelectronics, \\ Peking University, \\ Beijing, China
}

\begin{abstract}
This work described a system of high-speed data acquisition and real-time signal processing based on Tunable Diode Laser Spectroscopy (TDLS). We used wavelength modulation spectroscopy technique to improve the de-noising performance of the system and reduce the acquisition stress of the system. We specially designed a new structure of multi-channel TDLS, through the peripheral multi-channel Analog to Digital Converter (ADC) of the DSP (Digital Signal Processing) to measure multi-channel spectral information, which gives technical support for multi-component gas detection. We also compared the performance of the fixed point DSP with the float point DSP used in the TDLS system. By studying the aspects mentioned above, technical basis is provided for the implementation of standardized multi-channel TDLS system.
\end{abstract}

Keywords- TDLS; Second harmonic; Multi-channel; High-speed acquisition

\section{INTRODUCTION}

Tunable Diode Laser Spectroscopy (TDLS) is one of commonly used spectrum detection methods. Compared to other methods of spectrum measurement, TDLS has its unique advantages including fast response[1 3], high sensitivity[1], low cost[2,3], compact instrument design[3,4], far detection distance[5], and capability of testing on variety of pollutants at the same time[5,6]. These advantages of TDLS greatly promote its rapid development in a vast domain of applications[7,8]. In particular, TDLS has been widely applied in the detection of gas concentration[9,10] and the component of blood sugar as well as other substances.

In the TDLS system, the commonly method of data acquisition and signal processing is depicted as follow: With the data acquired by Analog to Digital Converter (ADC), computer was employed to analyze the result, from which the concentration of the gas was obtained[11]. In this process, the accuracy and the sampling speed of ADC is key point. In order to restore the waveform of the details, the sampling rate has to be much larger than the signal frequency.

In the TDLS system, each laser can only detect one kind of gas, while in complex applications multiple TDLS systems

\author{
D.M. Dong, L.Z. Jiao, P.C. Han, Y. Lang \\ National Engineering Research Center for Information \\ Technology in Agriculture \\ Beijing Academy of Agriculture and Forestry Sciences \\ Beijing 100097, China
}

which can simultaneously work for multi-component mixed gas detection are required. To the best of our knowledge, there has not been reported any systematic research on the implement method of the high-speed data acquisition and the real-time signal processing of multi-channel TDLS system.

Our paper including the following two parts: First, we discuss the high-speed data acquisition method of the directing absorption spectrum signal and the second harmonic signal in the TDLS system. Second, the signal processing performance of fixed point DSP and float point DSP in multi-channel TDLS system is compared. Such the research and analysis, will provide technical foundation to realize the standardized TDLS method.

\section{MATERIALS AND METHODS}

Our study has focused on three aspects: The laser signal driver module, detector signal processing module, and real-time signal sampling and processing module.

\section{A. Laser Driver and Photoelectric Detection System Design}

According to the law of Lambert-Beer, light intensity after absorption of the gas has a linear relationship with the gas concentration and the optical path[1]. Fig.1 shows the principle diagram of the multi-channel TDLS system. Laser 1 and laser 2 emit two different lasers, which will be detected by probe 1 and probe 2 after passing through the measured gas. M.E. Webber and Lundsberg-Nielsen had widely included the information of ammonia absorption peak position and strength in their paper, in which they proposed $1531 \mathrm{~nm}$ as the absorption peak of ammonia concentration detection and pointed out the advantages of this band was used for ammonia concentration detection[12-13]. Base on Hitran database and relate papers, we choose the $1650 \mathrm{~nm}$ as the absorption peak of methane. The laser used in our paper is manufactured by Chengdu Hua Yin photoelectric technology company, the ammonia DFB laser of 1531nm and the methane DFB laser of $1650 \mathrm{~nm}$. The probe will transmit the signal detected into detector signal processing module for processing. The photo-diode is TO type photo-diode that produced by Hai Te photoelectric Limited- Liability Company (LLC). As shown in 
Figure I, the laser driven signal is saw-tooth wave that is modulated by sine wave. The frequencies of the saw-tooth wave and sine wave we chose are $10 \mathrm{~Hz}$ and $5 \mathrm{KHz}$ respectively. The sine wave generated chip used is AD9850 and the saw-tooth wave generated chip used is DAC0832 and the main controller of this system is 51 Single Chip Micro-controller (SCM) in our paper.

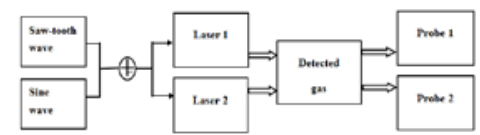

FIGURE I . LASER DRIVER AND PHOTOELECTRIC DETECTION SYSTEM SCHEMATIC DIAGRAM.

\section{B. Signal Processing System Design}

Figure II shows the principle diagram of the detector signal processing module of the multi-channel TDLS that our paper described. The two kinds of driver signal we used were saw-tooth wave signal and saw-tooth wave signal modulated by sine wave in the experiment. The theoretical basis of using these two kinds of signal is direct absorption spectroscopy technique and wavelength modulation spectroscopy technique.

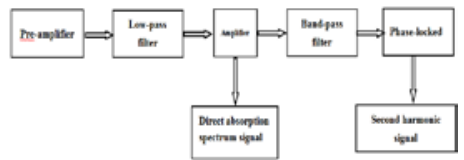

FIGURE II . THE PRINCIPLE DIAGRAM OF THE DETECTOR SIGNAL PROCESSING MODULE.

If the laser is driven by wavelength modulation spectroscopy technique, as shown in Figure II, due to the output of the probe is very weak, we must amplify the output of the probe using the pre-amplifier firstly, and then use the low-pass filter to acquire the low frequency signal which carrying concentration information. Meanwhile, the noise generated in the transfer process is filtered. Then we amplify the low frequency signal using the amplifier circuit. After that, the band-pass filter is used to acquire the second harmonic which carries concentration information but with higher amplitude [14]. Then the second harmonic signal is inputted to the phase-locked amplifier circuit, and the amplitude of the second harmonic will be obtained. If the laser is driven by direct absorption spectroscopy technique, we can get the direct absorption spectrum signal after filtering and pre-amplification.

In the part of signal acquisition, we used chip OPA277 as the main chip of the pre-amplifier circuit, chip OPA2277 as the main chip of the low-pass filter and the band-pass filter circuit, chip AD620 as the main chip of the amplifier circuit, and chip MLT04 as the main chip of phase-locked amplifier circuit .

\section{Real-Time Signal Acquisition System Design}

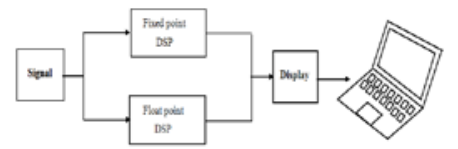

FIGUREIII. REAL-TIME SIGNAL PROCESSING SYSTEM.
FigureIII shows the real-time signal acquisition system. The signal in FigureIII is the output of the signal processing system. The signal will be sampled by DSP and calculate the concentration of the measured gas. The fixed DSP we used is TMS320F2812 and the float DSP we used is TMS320F28335, which were produced by TI. The information of gas concentration was real-time displayed by the Liquid Crystal Display (LCD), and we used 12864 LCD. During sampling process, we transmitted the data into computer for large data processing and analysis.

\section{Experiments}

Due to the limitations of the experimental conditions, we chose ammonia to verify the multi-channel TDLS system in the experiment.

We put $50 \mathrm{~mL}$ ammonia into the beaker the day before experiment, in which the concentration of ammonia was $12 \mathrm{~mol} / \mathrm{L}$. Then sealed the beaker to ensure a certain concentration of ammonia filled in the beaker. The next day we assembled the the multi-channel detection equipment that we designed, and inputted the saw-tooth wave to the laser driver circuit. Two of the same beaker were settled on the light path to collect a group of data, and another two beakers that filled with ammonia were put on the light path to collect the second group of data. After that, those two groups of data were transmitted into computer for further processing. With the second group of data subtracting the first group of data, it can avoid the breaker and air to impact on the curve of spectrum.

Then we inputted the wavelength modulation spectroscopy signal to the laser and collected data according to above methods. After that, we transmitted the collected data into compute to process and analyze.

\section{RESULTS AND ANALYSIS}

A. Multi-Channel Direct Absorption Spectrum Data Collection and Performance Analysis

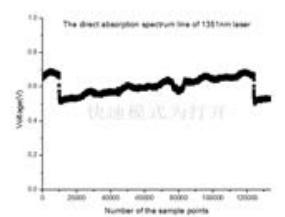

FIGURE IV. THE DIRECT ABSORPTION CURVE OF AMMONIA IN 1531NM.

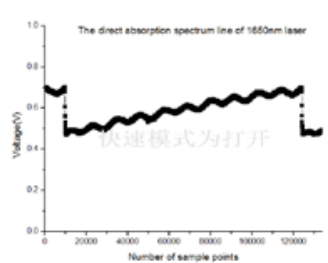

FIGURE V : THE DIRECT ABSORPTION CURVE OF AMMONIA IN 1650NM.

In our paper, one innovation is that we use two channel TDLS system to implement multi-component gas detection at the same time. FigureIV shows the direct absorption curve by 
using the $1531 \mathrm{~nm}$ laser to detect ammonia, from which we can see obvious absorption peak. Figure $V$ shows the direct absorption

curve by using 1650nm laser to detect ammonia, in which the ammonia absorbance is 0 in the $1650 \mathrm{~nm}[14]$. Therefore, ammonia has no absorption in the $1650 \mathrm{~nm}$ and no obvious absorption peak has been observed (Figure V). Such curve can test and verify the reliability of the multi-channel TDLS system.

Another innovation of our paper is high-speed data acquisition system. As shown in FigureIV and Figure $V$, we choose about 115000 points in each cycle of the experiment and the frequency of the saw-tooth laser driver signal is $10 \mathrm{~Hz}$, from which it can be seen that the sampling rate of our system can achieve $1.15 \mathrm{MHz}$. In previous studies, other system rarely showed such a high sampling rate, while our system has demonstrated high-speed data acquisition performance.

\section{B. Multi-Channel Data Analysis of Second Harmonic Signal}

From previous study we can know that the first harmonic does not contain the concentration information, while second harmonic contains the concentration information[14]. So the concentration of measured gas can be calculated by the ratio of first harmonic and second harmonic, which can relieve the pressure of the system on sampling. Figure VI shows the second harmonic absorption curve of ammonia in 1531nm and Figure VII shows the second harmonic absorption curve of ammonia in $1650 \mathrm{~nm}$. As the absorbance of ammonia is 0 at 1650nm [14], the curve in FigureVII shows a straight line and the amplitude should be $0 \mathrm{~V}$. But the amplitude shown in FigureVII is $0.1 \mathrm{~V}$, which should be attributed to the error of the sampling system. The accuracy of system sampling is determined by the $\mathrm{A} / \mathrm{D}$ sampling bit, which has nothing to do with the performance of our system, and will be mentioned in the latter part.

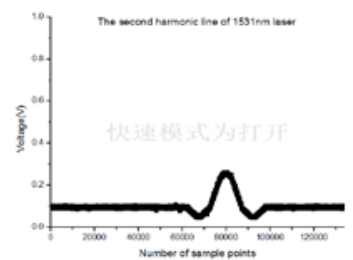

FIGUREVI. THE SECOND HARMONIC CURVE OF AMMONIA IN 1531NM.

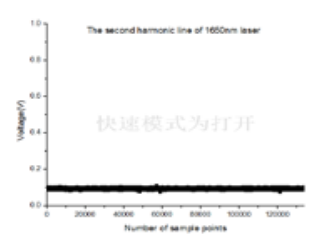

FIGUREVII. THE SECOND HARMONIC CURVE OF AMMONIA IN $1650 \mathrm{NM}$.

The direct absorption spectrum is vulnerable interfered by the background noise, so the curve of the spectral line is coarse, as the FigureIV and Figure $V$ shown. But the second harmonic overcome the difficulty effectively, which can effectively remove the background noise on the influence of spectral lines. As shown in the FigureVI and FigureVII, the curve of the second harmonic is smooth. It is known that the wavelength modulation spectroscopy technique is more effective than the direct absorption spectrum technique to reducing the noise, which could increase the signal-to-noise ratio.

\section{Performance Analysis of Real-Time Processing System}

In order to verify the superiority of the DSP that we choose in our system, we select another fixed point DSP TMS320F2812. In the experiment, we selected 6 groups of different data that have been processed by computer, and did Fast Fourier Transform (FFT) for these data using the two kinds of DSP respectively, and recorded the running time of the system shown in Table I. In terms of the sampling accuracy, the system sampling quantization precision is only related to the precision of $\mathrm{A} / \mathrm{D}$ chip, which is barely related to the selection of the DSP[15]. In the digital signal processing system, due to data storage, operation, the different data storage format and large data, the fixed point DSP will generate the system sampling accuracy loss and overflow, but the float point DSP does not happen. The methods of double word storage scaling and block floating point are usually used to overcome the data large in fixed-point arithmetic, however, which is at the cost of losing storage, wasting of CPU execution time and increasing the difficulty of software developing. The float point DSP shows its unique advantages, which can guarantee to achieve high precision complex algorithm and real-time signal processing system.

TABLE I . THE SYSTEM RUNNING TIME OF USING FIXED POINT DSP AND FLOAT POINT DSP.

\begin{tabular}{|l|l|c|c|c|c|c|c|}
\hline \multicolumn{2}{|c|}{ Sampling Points } & 16 & 32 & 64 & 128 & 256 & 512 \\
\hline $\begin{array}{l}\text { Run } \\
\text { ning }\end{array}$ & TMS320F & 1.01 & 2.35 & 5.32 & 11.8 & 26.1 & 57.0 \\
Time & 2812 & & & & 5 & 2 & 3 \\
\cline { 2 - 8 } & TMS320F & 0.99 & 2.32 & 5.34 & $\begin{array}{c}11.8 \\
4\end{array}$ & $\begin{array}{c}26.0 \\
5\end{array}$ & $\begin{array}{c}44.8 \\
8\end{array}$ \\
\hline
\end{tabular}

From the analysis of the above, we can know that the float point DSP we chose in our system can provide high precision data operation and simple software design patterns without losing time advantage. This will improve the performance of our system.

\section{CONCLUSIONS}

In conclusion, through the analysis above several advantages of the system can be observed: high-speed data acquisition system improves the overall performance of the system; the wavelength modulation spectroscopy technique releases the sampling suppress and improves the de-noising performance of the system; and the superiority and the reliability of the multi-channel detection improves the performance of the system. Our work will provide strong technical support for the gas detection equipment research and development.

\section{ACKNOWLEDGMENTS}

This work was supported by Beijing Natural Science Foundation (No. 4131002). 


\section{REFERENCES}

[1] KAN Rui-Feng, DONG Feng-Zhong, ZHANG Yu-Jun, LIU Jian-Guo, LIU Cheng, WANG Min, GAO Shan-Hu, CHEN Jun, "Influence of the laser intensity in second-harmonic detection with tunable diode laser multi-pass absorption spectroscopy: comparison of experiment and theory”. Chinese Physics, 14(9) pp.1904-1909,2005.

[2] LiFei,YU Xi-long,CHEN Li-hong,ZHANG Xin-yu. Temperature and water vapor concentration measurements of $\mathrm{CH} 4 /$ Air premixed flat flame based on TDLAS. Journal of Experiments in Fluid Mechanics. 23(2),pp.40-44,2009.

[3] ZHANG Zhuo1, ZHANG Li-ying2, TDLAS-based methane telemetry technology.JOURNAL OF CHANGCHUN UNIVERSITY, 21(6),pp.9-12,2011.

[4] WANG Tie-dong*, LIU Wen-qing, ZHANG Yu-jun, LIU Jian-guo, DONG Feng-zhong, WANG Min, WANG Xiao-mei, XU Min. Romoto Sensing CO,CO2 in Vehicle Emissions Based on TDLAS.Laser Technology \& Applications,05,pp.25-30,2007.

[5] Li Sheng1, Xiao Bing2. Measurment of CO2 Dynamic Concentration and Temperature Based on TDLAS. Automation \& Information Engineering. 04,pp.8-11,2006.

[6] Guo Huafang, Li Junhong, Hu Yueming, Zeng Jun. A portable remote $\mathrm{CO}$ and $\mathrm{CO} 2$ sensor us ing a tunable diode laser. Microcompute information,22(5-1),pp.148-150,2006.

[7] S.Wagner, B.T.Fisher, J.W.Fleming, et al. TDLAS-based in situ measurement of absolute acetylene concentrations in laminar 2D diffusion flames. Processings of the Combustion Institute, 32(1),pp.839-846,2009.

[8] A.Farooq,J.B.Jeffries and R.K.Hanson. CO2 concentration and temperature sensor for combustion gaser using diode-laser absorption near $2.7 \mu \mathrm{m}$. Applied Physics B, 90(3-4),pp.619-618,2008.

[9] KAN Rui-feng, Liu Wen-qing, ZHANG Yu-jun, et al. Tunable diode laser absorption spectrometer monitors the ambient methane with high sensitivity. Chinese Journal of Laser, 32(9),pp.1217-1220,2005.

[10] Wang J,Maiorov M,Hanson R K, et al.In situ combustion measurement of CO with diode-laser absorption near $2.3 \mu \mathrm{m}$. Applied Optics, 39(30),pp.5579-5589,2000.

[11] WANG Jian1,2**, HUANG Wei2, GU Hai-tao1,2, LI Ying2, LIU Li-peng2, GAO Xiu-min1,2. Gas Temperature Measure Based on TDLAS. Journal of Optoelectronics Laser, 17(10),pp.1233-1237,2006.

[12] Michael.E.Webber. Diode laser measurements of NH3 and $\mathrm{CO} 2$ for combustion and bioreactor applications. Stanford: Stanford University ,pp.13-27,2001.

[13] Michael.E.Webber, Douglas S.Baer, and Ronald K.Hanson. Ammonia monitoring near $1.5 \mu \mathrm{m}$ with diode-laser absorption sensors. Applied Optics,40(12),pp.2031-2042,2001.

[14] Zhao Zhi.Research on Ammonia Detection Based on Tunable Diode Laser Spectroscopy Sensing Technique.Beijing Jiaotong University, 2011.

[15] ZHAO Sheng-kai,QIU Kuan-min. Development of DSP and Comparison Between Fixed Point and Float Point of Process Method. JOURNAL OF NORTHERN JIAOTONG UNIVERSITY,24(05)pp.82-88,2000. 